\title{
EM interaction of handset antennas and a human in personal communications
}

Michael A. Jensen

jensen@byu.edu

Yahya Rahmat-Samii

Follow this and additional works at: https://scholarsarchive.byu.edu/facpub

Part of the Electrical and Computer Engineering Commons

\section{Original Publication Citation}

Jensen, M. A., and Y. Rahmat-Samii. "EM Interaction of Handset Antennas and a Human in Personal Communications." Proceedings of the IEEE 83.1 (1995): 7-17

\section{BYU ScholarsArchive Citation}

Jensen, Michael A. and Rahmat-Samii, Yahya, "EM interaction of handset antennas and a human in personal communications" (1995). Faculty Publications. 692.

https://scholarsarchive.byu.edu/facpub/692

This Peer-Reviewed Article is brought to you for free and open access by BYU ScholarsArchive. It has been accepted for inclusion in Faculty Publications by an authorized administrator of BYU ScholarsArchive. For more information, please contact ellen_amatangelo@byu.edu. 


\title{
EM Interaction of Handset Antennas and a Human in Personal Communications
}

\author{
MICHAEL A. JENSEN, STUDENT MEMBER, IEEE, AND YAHYA RAHMAT-SAMII, FELLOW, IEEE
}

In personal communications, the electromagnetic interaction between handset-mounted antennas and the nearby biological tissue is a key consideration. This paper presents a thorough investigation of this antenna-tissue interaction using the finite-difference time-domain (FDTD) electromagnetic simulation approach with detailed models of real-life antennas on a transceiver handset. The monopole, side-mounted planar inverted $F$, top-mounted bent inverted $F$, and back-mounted planar inverted $F$ antennas are selected as representative examples of external and internal configurations. Detailed models of the human head and hand are implemented to investigate the effects of the tissue location and physical model on the antenna performance. Experimental results are provided which support the computationally obtained conclusions. The specific absorption rate (SAR) in the tissue is examined for several different antenna/handset configurations. It is found that for a head-handset separation of $2 \mathrm{~cm}$, the $S A R$ in the head has a peak value between 0.9 and $3.8 \mathrm{~mW} / \mathrm{g}$ and an average value between 0.06 and $0.10 \mathrm{~mW} / \mathrm{g}$ for $1 \mathrm{~W}$ of power delivered to the antenna. Additionally, the head and hand absorb between 48 and $68 \%$ of the power delivered to the antenna.

\section{INTRODUCTION}

The introduction of cellular communications technology has generated a widespread awareness of the important role wireless services play in today's communications-centered marketplace [1]. Naturally, this awareness has spawned a growth in the wireless communications arena which in turn has had and will continue to have an impact on antenna technology advancement. An important example of this influence involves the development of external and internal antenna structures, such as the monopole or planar inverted $F$ antenna (PIFA), which can be efficiently and conveniently integrated with a hand-held transceiver unit. The dominant role of such portable terminals in many systems motivates a thorough examination of the issues involved in designing these and other similar antenna structures.

Because portable handsets operate in close proximity to a human being, one particularly important consideration involves the interaction of the radiated electromagnetic fields with the nearby biological tissue. Certainly, the operator's influence on the antenna gain, radiation pattern, and input impedance is an issue which deserves a detailed investiga-

Manuscript received February 22, 1994; revised August 30, 1994. This work was supported by ARPA Contract Number DAAB07-93-C-C501.

The authors are with the Electrical Engineering Department, University of California at Los Angeles, Los Angeles, CA 90024-1594 USA

IEEE Log Number 9406485. tion. Furthermore, growing concerns over the health effects of tissue exposed to electromagnetic energy motivates an effort to understand the power absorption distribution in the tissue when a hand-held device is used.

To gain a detailed understanding of the antenna-tissue interaction, the designer must abandon simplified, approximate analysis methodologies in favor of more general numerical simulation techniques. The finite-difference timedomain (FDTD) method [2]-[6] is one such approach which allows accurate prediction of the electromagnetic behavior of general topologies. A few studies have appeared which present preliminary results associated with this topic. For example, one paper has used a homogeneous sphere and block of muscle to represent the head and hand respectively, within an FDTD simulation of a handsetmounted monopole antenna [7]. Other studies have used a more elaborate model of the head, but have used simpler models for the antenna such as a dipole [8].

In this work, advanced physical models of the head and hand are coupled with detailed representations of handsetmounted antennas to allow accurate FDTD simulations of real-life communications scenarios. The computer program developed for this work has been made adequately flexible to accommodate these modeling requirements. The computations not only reveal the human operator influence on the antenna performance, but also provide information concerning the SAR [8]-[10] for power absorbed in the tissues. The monopole, side-mounted PIFA, top-mounted bent inverted $F$ antenna (BIFA), and back-mounted PIFA elements shown in Fig. 1 are used in the computational examples to provide results representative of external and internal antennas. The effect of tissue location and the model used to represent the human operator are addressed. Measured data is compared to many of the computational results to show the accuracy of the FDTD tool in predicting the antenna performance. Conclusions and suggestions are presented based upon the results obtained.

\section{PHYSICAL/COMPUTER MODELING}

\section{A. FDTD Implementation}

The FDTD methodology [2]-[10] is derived from Maxwell's time-domain equations which may be expressed 


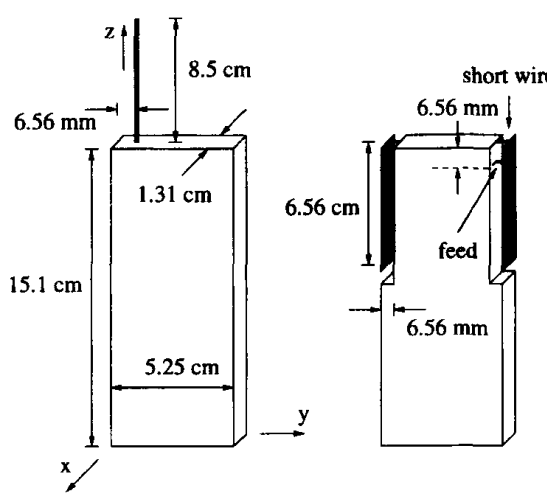

(a) (b)

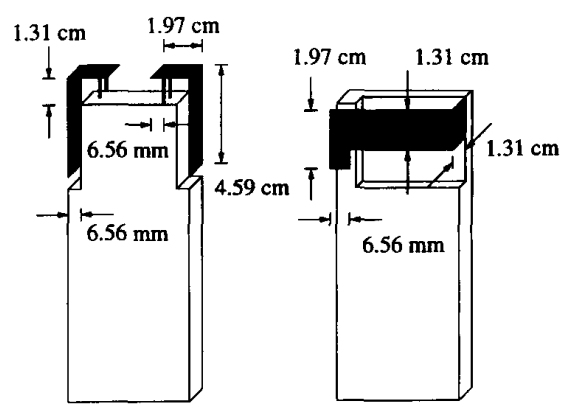

(c)

(d)

Fig. 1. Antenna geometries and dimensions for hand-held transceiver units: (a) monopole; (b) side-mounted dual PIFA; (c) top-mounted dual BIFA; (d) back-mounted PIFA. The chassis dimensions $\left(105 \mathrm{~cm}^{3}\right)$ shown in (a) apply to all four configurations.

as

$$
\begin{aligned}
\mu \frac{\partial \vec{H}}{\partial t} & =-\nabla \times \vec{E} \\
\epsilon \frac{\partial \vec{E}}{\partial t} & =\nabla \times \vec{H}-\sigma \vec{E}
\end{aligned}
$$

where $\vec{E}$ and $\vec{H}$ are the electric and magnetic field intensities and $\epsilon, \mu$, and $\sigma$ are the space-dependent permittivity, permeability, and conductivity, respectively. Using a discretization of the calculus operators in these equations results in a set of algebraic time-stepping relations which may be written in a compact form as

$$
\begin{aligned}
\vec{H}^{n+1 / 2}= & \vec{H}^{n-1 / 2}+\overline{\bar{\gamma}} \cdot\left\{D_{x}\left(-\hat{x} \times \vec{E}^{n}\right)\right. \\
& \left.+D_{y}\left(-\hat{y} \times \vec{E}^{n}\right)+D_{z}\left(-\hat{z} \times \vec{E}^{n}\right)\right\} \\
\vec{E}^{n+1}= & \overline{\bar{\alpha}} \cdot \vec{E}^{n}+\overline{\bar{\beta}} \cdot\left\{D_{x}\left(\hat{x} \times \vec{H}^{n+1 / 2}\right)\right. \\
& \left.+D_{y}\left(\hat{y} \times \vec{H}^{n+1 / 2}\right)+D_{z}\left(\hat{z} \times \vec{H}^{n+1 / 2}\right)\right\}
\end{aligned}
$$

where the superscript denotes the time step $t=n \Delta t$ and the difference operator is defined as

$$
D_{x} \vec{f}(x, y, z)=\frac{\vec{f}(x+\Delta x / 2, y, z)-\vec{f}(x-\Delta x / 2, y, z)}{\Delta x} .
$$

The terms $\overline{\bar{\alpha}}, \overline{\bar{\beta}}$, and $\overline{\bar{\gamma}}$ are space-dependent diagonal tensors whose components are defined by

$$
\alpha_{p p}=\frac{\frac{\bar{\epsilon}_{p}}{\Delta t}-\frac{\bar{\sigma}_{p}}{2}}{\frac{\bar{\epsilon}_{p}}{\Delta t}+\frac{\bar{\sigma}_{p}}{2}} \quad \beta_{p p}=\frac{1}{\frac{\bar{\epsilon}_{p}}{\Delta t}+\frac{\bar{\sigma}_{p}}{2}} \quad \gamma_{p p}=\frac{\Delta t}{\bar{\mu}_{p}}
$$

for $p=x, y$, or $z$. The symbols characterized by an overbar $(\cdot)$ represent averaged values of the constitutive parameters over a face of a cell in the discretized computational grid, such as

$$
\bar{\epsilon}_{p}=\frac{\int_{\Delta S} \epsilon(\vec{r}) \hat{p} \cdot d \vec{S}}{\Delta S}
$$

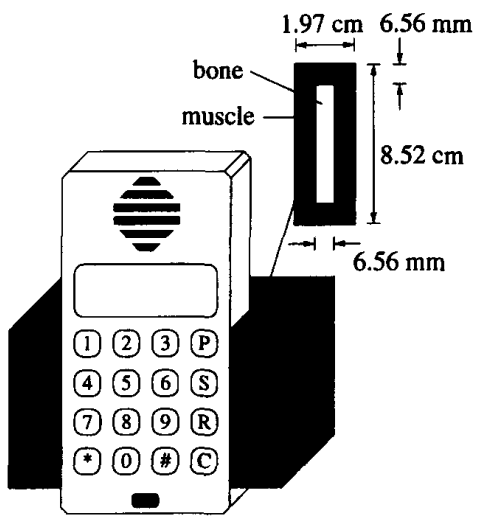

Fig. 2. Block model of the human hand used in the simulation of antennas on the handset.

where $\Delta S$ represents the area of the cell face normal to the unit vector $\hat{p}$. Using the definitions in (6) and (7) allows accurate modeling of inhomogeneous materials within the computational domain.

The relations in (3) and (4) are used to track the timeevolution of the fields in the spatial domain by initially setting all field values to zero. An antenna excitation is then introduced by specifying a voltage at the antenna feed point. The value of the magnetic and electric field intensities are alternately computed at times $t=(n+1 / 2) \Delta t$ and $t=(n+1) \Delta t$. At grid points coincident with perfect electric conductors, the tangential components of $\vec{E}$ are set to zero at each time step. Special considerations are made for modeling wires and lumped elements within the domain [5], and absorbing boundary conditions [11] are used to truncate the mesh a reasonable distance from the antenna. Once the time-domain data has been collected, a Fourier transform is used to obtain the desired frequency-domain quantities such as input impedance, radiation pattern, and gain. In this fashion, the antenna response over a given bandwidth may be obtained.

PROCEEDINGS OF THE IEEE, VOL. 83, NO. 1, JANUARY 1995 


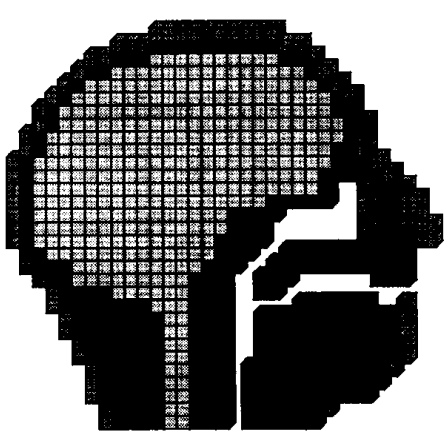

(a)

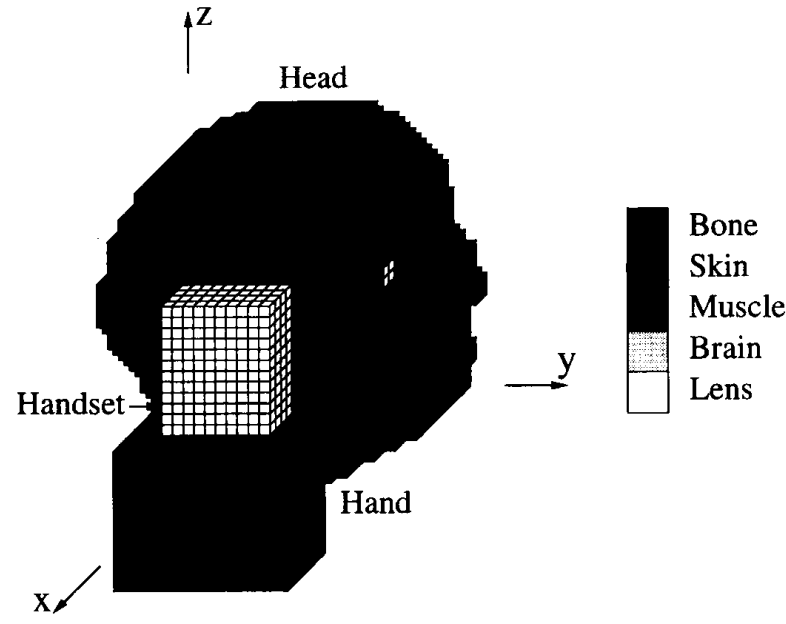

(b)

Fig. 3. (a) Sagittal cut of the discrete head model at the head center and (b) entire head model with the hand and plastic-covered handset present.

The antenna excitation is introduced into the computational domain using a previously reported simulated coaxial feed model [5]. The functional form of the voltage applied in the feed is a sinusoid modulated by a Gaussian as expressed by

$$
s(t)=e^{-\left(t-t_{o}\right)^{2} / 2 \sigma_{t}^{2}} \cos \left[2 \pi f_{o}\left(t-t_{o}\right)\right]
$$

where $f_{o}$ is the sinusoidal frequency and $\sigma_{t}$ controls the pulse width. This particular functional form is interesting since the center frequency and bandwidth of its spectrum may be controlled. In this work, antenna impedance computations over a band are performed using the parameters $\sigma_{t}=$ $160 \mathrm{ps}$ and $f_{o}=0$. In computing this transient response, the time-stepping is continued until the antenna current magnitude has decayed to a level approximately $40 \mathrm{~dB}$ below its peak value. When computing radiation patterns and power absorption in tissues, a single frequency source is used such that $\sigma_{t} \rightarrow \infty$. In this case, the time-stepping is performed for several cycles $(\sim 20)$ in order for the system to reach a steady state.

\section{B. Biological Tissue Modeling}

Anatomical human features are modeled within the FDTD framework by mapping the spatial location of the different tissues into a permittivity and conductivity assignment in the computational grid. The human hand is simply modeled as a layer of bone surrounded by a layer of muscle that covers three sides of the handset, as depicted in Fig. 2. To construct a head model, a grid with a $6.56 \mathrm{~mm}$ spatial resolution was placed on cross-sectional images of the head obtained from an anatomy atlas [12]. Magnetic resonance images (MRI) were also used to aid in the tissue classification and location. Each cell in the grid was then assigned a permittivity and conductivity classification corresponding to the type of tissue which filled the majority of the cell. Figs. 3(a) and (b) illustrate a mid-sagittal cross section of the head model and the full head model ( 24 $\times 33 \times 31$ cells) with the hand and handset. Fig. 4 provides two different views of the head/hand/handset configuration with the dimensions used in the computations. The electrical parameters corresponding to each tissue around the operating frequency of $915 \mathrm{MHz}$ have been obtained from published data and are listed in Table 1 [9]. Since variations in the conductivity and permittivity values are less than $5 \%$ and $1 \%$, respectively, over the frequency band of interest, these constitutive parameters will be considered to be nondispersive in the FDTD simulations. If broadband computations are to be performed where the dispersive nature of the tissues is more significant, then special considerations in the FDTD implementation must be made [13].

Two additional comments are warranted concerning the tissue models presented. First, in order to allow the modeling of very realistic operator/handset configurations, it is important to allow the handset to be rotated such that it is positioned between the operator's mouth and ear. This is accomplished by rotating the position of each cell in the head model in the $y-z$ plane about the head center and reconstructing the grid based upon these rotated tissue locations (see Fig. 4). The fact that the handset remains aligned with the FDTD grid allows accurate modeling of its rectilinear features. Second, when using the models in the computations, different FDTD cell sizes are chosen according to the parameter of interest. For example, it has been found that a cell size of $3.28 \mathrm{~mm}$ is required in order to obtain an accurate value of the input impedance. However, for pattern and power absorption characterization, a $6.56 \mathrm{~mm}$ cell size may be used. These two cell sizes correspond to approximately 14 and 7 cells per wavelength, 
Table 1. Relative Permittivity, Conductivity, and Density of the Tissues in the Hand and Head Near $900 \mathrm{MHz}$

\begin{tabular}{lccc}
\hline Tissue & Permittivity & Conductivity (S/m) & $\begin{array}{l}\text { Density } \\
\left(\mathrm{g} / \mathrm{cm}^{3}\right)\end{array}$ \\
\hline Bone & 8.0 & 0.105 & 1.85 \\
Skin/Fat & 34.5 & 0.60 & 1.10 \\
Muscle & 58.5 & 1.21 & 1.04 \\
Brain & 55.0 & 1.23 & 1.03 \\
Humour & 73.0 & 1.97 & 1.01 \\
Lens & 44.5 & 0.80 & 1.05 \\
Cornea & 52.0 & 1.85 & 1.02 \\
\hline
\end{tabular}
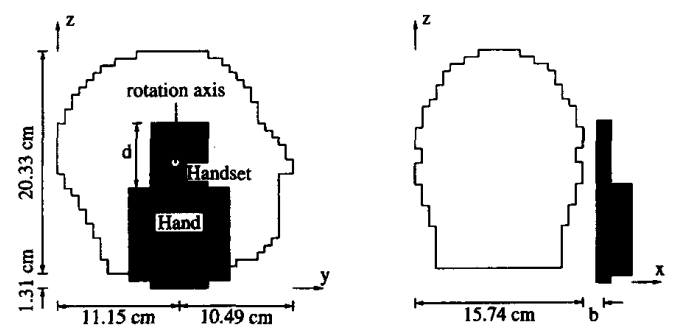

Fig. 4. Side and rear views of the FDTD head/hand/handset model showing dimensions.

respectively, within the high-permittivity tissues at the operating frequency.

\section{Time-Response of High-Permittivity Dielectrics}

The relatively high permittivity values listed in Table 1 suggest that a considerable amount of computational time (i.e., large number of time steps) is required for an antenna near a biological system to reach its steadystate response for a given input. To test the duration of the transient effects, a $16.4 \mathrm{~cm}$ dipole is placed $1.31 \mathrm{~cm}$ from a $17 \mathrm{~cm}$ diameter homogeneous dielectric sphere. The antenna is excited with a 160 ps Gaussian pulse and the antenna terminal current $I_{s}$ is monitored as a function of time for different values of the sphere permittivity and conductivity. Fig. 5 illustrates the result of this study, where the normalization parameters $\eta_{o}$ and $c_{o}$ are the free-space impedance and speed of light, respectively. The dielectric parameters are chosen to represent the humor in the eye since this is the highest-permittivity tissue involved in the models. As can be seen, introduction of high-permittivity dielectrics produces significant ringing in the antenna response. However, addition of loss into the system causes the transient response to damp quickly. The response for the dipole near the head model also shown in Fig. 5 illustrates that this same damping effect occurs for the inhomogeneous tissue models used in this investigation.

\section{Power Absorption and SAR}

Several quantities of interest should be defined before proceeding with the results. The power absorbed within the

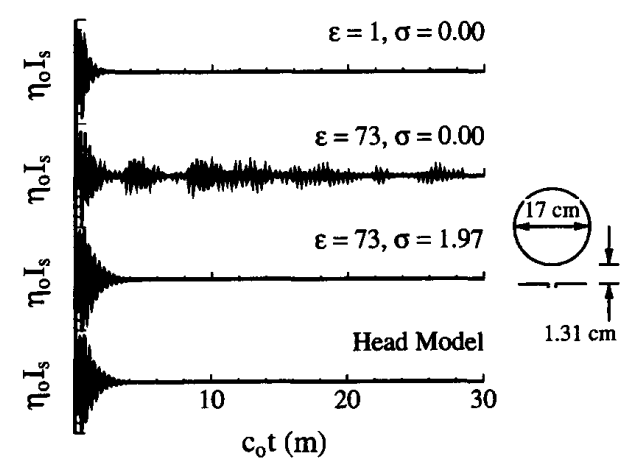

Fig. 5. Normalized antenna current versus normalized time for a dipole $1.31 \mathrm{~cm}$ from a homogeneous sphere filled with dielectrics of different permittivity and conductivity values. The case for a dipole next to the inhomogeneous head model is also provided.

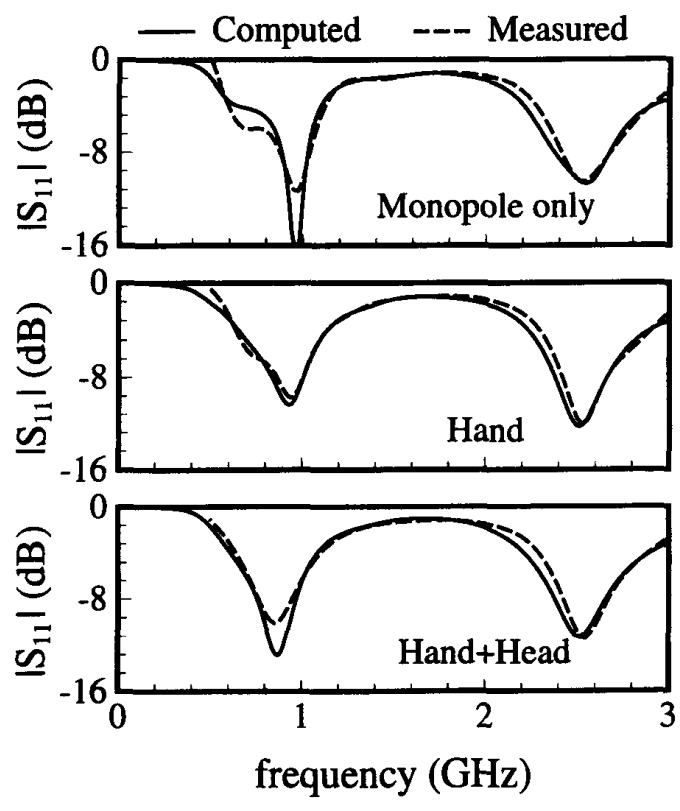

Fig. 6. Computed and measured values of $\left|S_{11}\right|$ for the monopole on the handset with no tissue, with the hand only, and with the hand and head $(b=1.97 \mathrm{~cm}, d=5.9 \mathrm{~cm})$.

lossy tissue $\left(P_{a b s}\right)$ and the power radiated to the far-field $\left(P_{\text {rad }}\right)$ are obtained using the integrals

$$
\begin{aligned}
& P_{\mathrm{abs}}=\frac{1}{2} \int_{V} \sigma|\mathbf{E}|^{2} d V \\
& P_{\mathrm{rad}}=\frac{1}{2} \operatorname{Re}\left\{\int_{S} \mathbf{E} \times \mathbf{H}^{*} \cdot \hat{n} d S\right\}
\end{aligned}
$$

where $\mathbf{E}$ and $\mathbf{H}$ are the frequency domain peak electric and magnetic fields, respectively, and $\sigma$ is the medium conductivity. The symbols $V, S$, and $\hat{n}$, represent the volume containing the tissue, the surface completely surrounding the handset/operator configuration, and the unit outward normal to the surface, respectively. The antenna/tissue 

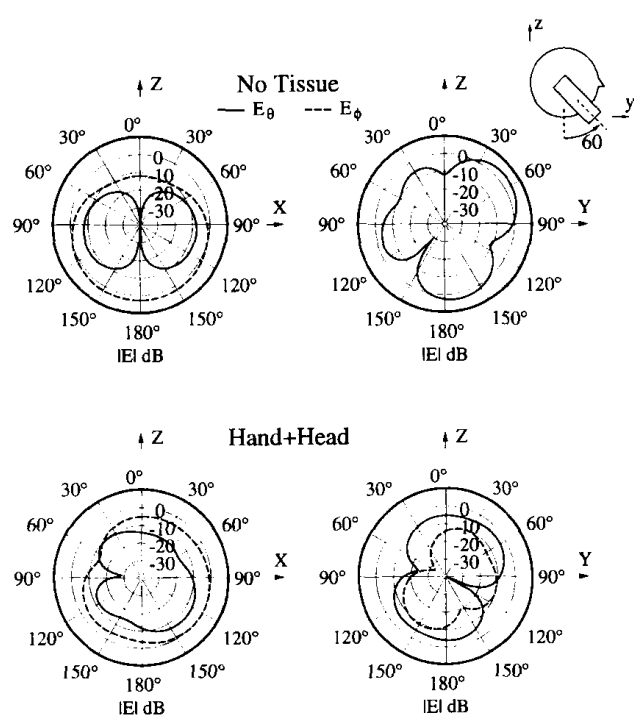

Fig. 7. Computed radiation patterns at $915 \mathrm{MHz}$ normalized to the antenna gain for a monopole on the plastic-covered handset in the absence and presence of the head and hand $(b=1.97 \mathrm{~cm}, d=$ $5.9 \mathrm{~cm}$ ). The antennas are rotated $60^{\circ}$ from upright.

system radiates with an efficiency of

$$
\eta_{a}=\frac{P_{\mathrm{rad}}}{P_{\mathrm{rad}}+P_{\mathrm{abs}}}=\frac{P_{\mathrm{rad}}}{P_{\mathrm{del}}}
$$

where $P_{\text {del }}$ is the real power delivered to the antenna.

The SAR quantifies the power absorbed per unit mass of tissue and is a fundamental parameter when discussing the health risks of electromagnetic power absorption in the body. This quantity is defined as

$$
\mathrm{SAR}=\frac{\sigma}{2 \rho}|\mathbf{E}|^{2}
$$

where $\rho$ is the material density. The values of $\sigma$ and $\rho$ for the different tissue types are provided in Table 1 [9]. The ANSI/IEEE standard suggests that the SAR averaged over any $1 \mathrm{~g}$ of tissue for $30 \mathrm{~min}$. or more should remain under $1.6 \mathrm{~mW} / \mathrm{g}$ [14], [15]. If necessary, the SAR values can be manipulated to obtain the tissue heating resulting from the power dissipation through a simple multiplicative constant.

\section{COMPUTATIONAL AND EXPERIMENTAL RESUltS}

The following cases provide examples of computations and measurements used in the evaluation of several antenna/handset configurations. The experimental measurements of $S_{11}$ provided here have been obtained from a Hewlett-Packard 8510B network analyzer at the University of California, Los Angeles, antenna measurement facility. This parameter is a measure of the reflection coefficient for a voltage wave introduced to the antenna feed point using a $50 \Omega$ coaxial cable and may be defined as

$$
S_{11}=\frac{Z_{a}-50}{Z_{a}+50}
$$

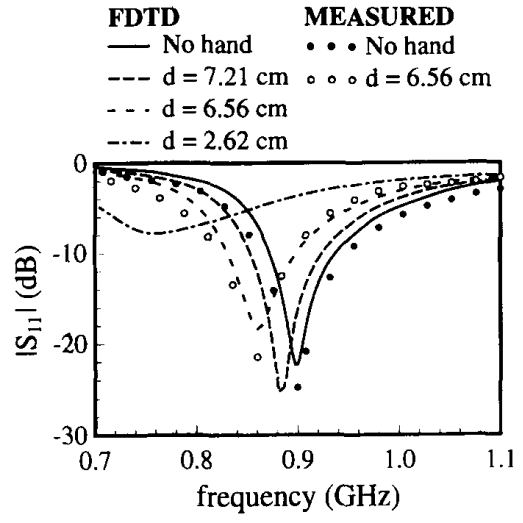

Fig. 8. Computed value of $\left|S_{11}\right|$ for the side-mounted PIFA on the handset without the hand and with the hand for three different values of $d$ (see Fig. 4). Measured values appear for the configurations with no hand and with the hand at $d=6.56 \mathrm{~cm}$.

where $Z_{a}$ is the antenna input impedance. For all of the examples, the feeding wire radius is $r_{o}=0.46 \mathrm{~mm}$ which corresponds to the inner conductor radius of RG402/ $50 \Omega$ semi-rigid coaxial cable. Where impedance measurements involving biological tissue are presented, a single human subject is used. These experiments have been deliberately performed at very low power levels such that induced SAR levels are well below recommended safety limits.

The computer platform used in the simulations is an IBM RISC/6000 $530 \mathrm{H}$ workstation. Based upon the findings of Section II-C, the simulations for the small (large cell size) and large (small cell size) models require 1800 and 3600 time steps, respectively, corresponding to respective physical run times of approximately $2-3$ hours and 10-12 hours. The storage requirements are approximately $17 \mathrm{MB}$ for the small model and $35 \mathrm{MB}$ for the large model.

The geometries and dimensions for the different antennas under investigation are illustrated in Fig. 1. Parts (a)-(d) illustrate the monopole, side-mounted PIFA, top-mounted BIFA, and back-mounted PIFA configurations, respectively. The chassis dimensions provided in Fig. 1(a) apply to all four geometries. When the plastic casing is included, it is modeled as a $3.28 \mathrm{~mm}$ thick lossless dielectric with a relative permittivity of 2 which is immediately adjacent to and completely surrounds the chassis. In computations where the FDTD cell size is larger than $3.28 \mathrm{~mm}$, the approach detailed in [16] is used to approximate the thin plastic casing.

\section{A. Monopole Antenna}

The monopole antenna's widespread use in the personal wireless industry motivates an investigation of its characteristics and performance. The geometry for the quarter-wave monopole antenna on the conducting handset chassis is shown in Fig. 1(a). Fig. 6 presents the value of $\left|S_{11}\right|$ versus frequency for this configuration obtained both computationally and experimentally for the handset alone, the handset with the hand $(d=5.9 \mathrm{~cm})$, and the 


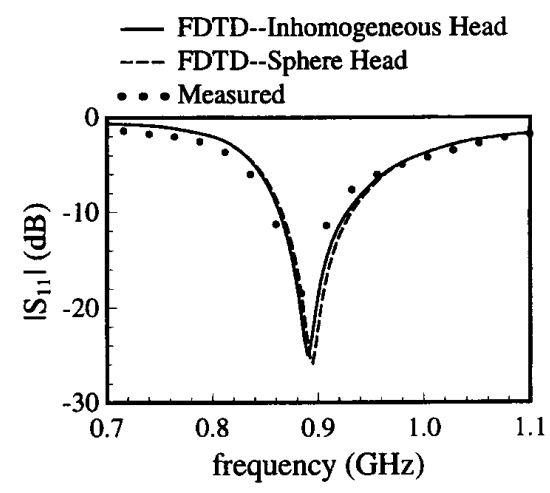

Fig. 9. $\left|S_{11}\right|$ versus frequency for the side-mounted PIFA on the handset with the head and the hand $(b=1.97 \mathrm{~cm}, d=7.21 \mathrm{~cm})$. Computed results obtained using both head models are compared with measured data.

handset with the hand and the head $(b=1.97 \mathrm{~cm})$. For simplicity, the data for the third case is obtained with the handset oriented upright with respect to the head, as illustrated in Fig. 4. Because no plastic casing was included in the experimental handset prototype, the casing was absent in the computations as well. As can be seen, the FDTD method is very effective in predicting the antenna impedance behavior. Furthermore, the results imply that the hand and head exercise relatively little influence on the antenna input impedance. This is an important feature of the monopole which partly accounts for its widespread use.

The monopole's radiation pattern, in contrast to its input impedance, is more noticeably influenced by the operator proximity. This is demonstrated in Fig. 7 which provides the radiation pattern of the handset-mounted monopole at $915 \mathrm{MHz}$ in the absence and presence of the hand and head. In this simulation, the plastic-covered handset is rotated $60^{\circ}$ in the $y-z$ plane with respect to the head (see figure inset), and the patterns are normalized to the antenna gain. These patterns are presented in standard spherical coordinates such that the principal polarizations are in the $\hat{\theta}$ and $\hat{\phi}$ directions. Clearly, the presence of the biological tissue alters the radiation pattern and reduces the antenna gain. The ratio of the power absorbed in the head and hand to the total power delivered to the antenna, as well as the antenna radiation efficiency, are provided in Table 2 for the 915 $\mathrm{MHz}$ operation frequency. This table contains data for the handset rotated as in Fig. 7 and for the handset upright as shown in Fig. 4. As can be seen, the results are very similar for the two handset orientations. The gain reduction caused by power absorption is a key issue to consider in determining system requirements to satisfy target link budgets.

\section{B. Side-Mounted PIFA}

The growing desire to replace the monopole with more conformal, less obtrusive elements has focussed considerable attention on antennas such as the PIFA [17]. A potential topology with two PIFA elements configured
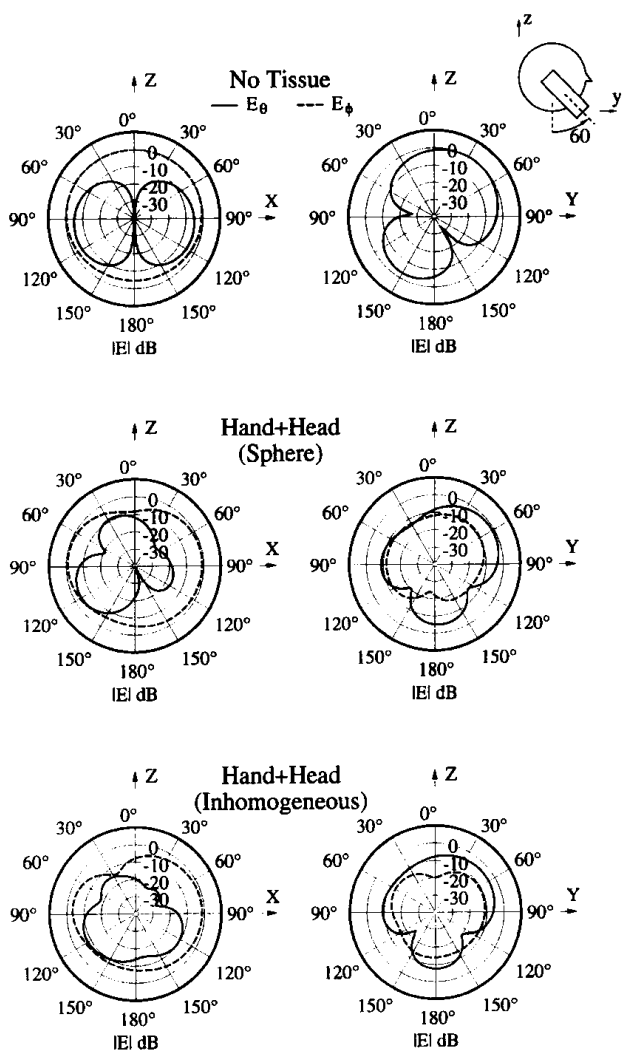

Fig. 10. Computed patterns at $915 \mathrm{MHz}$ normalized to the antenna gain for the side-mounted PIFA on the plastic-covered handset rotated $60^{\circ}$ from upright. Results are shown for the handset alone, with the spherical head model, and with the inhomogeneous head model $(b=1.97 \mathrm{~cm}, d=7.21 \mathrm{~cm})$.

to provide dual-branch antenna diversity is illustrated in Fig. 1(b) [5]. These side-mounted elements consist simply of a probe-fed conducting plate suspended above the conducting chassis. A short circuiting wire or strip is attached to one end of the suspended plate- a configuration which allows considerable reduction in the element resonant size. The antenna can generally be matched to a $50 \Omega$ feeding line through proper selection of the feed point, shorting pin location, and antenna dimensions.

Fig. 8 illustrates the value of $\left|S_{11}\right|$ versus frequency for one PIFA element with the second terminated in a matched load for several handset/operator configurations. The curves compare the results when no tissue is included to those when the hand is placed at three vertically displaced positions on the handset. Once again, no plastic casing is included in order to allow comparison with measured results. As can be seen from the plot, the hand position exercises a detuning effect on the antenna resonant frequency and impedance. Most significant is the high impedance mismatch which occurs when the hand begins to mask the antenna. These results illustrate the importance of minimizing antenna masking through proper antenna placement. The dots in the figure indicate experimentally 
Table 2. Computed Normalized Power Absorption and Peak SAR (mW/g/W) in the Head and Hand, Average SAR $(\mathrm{mW} / \mathrm{g} / \mathrm{W})$ in the Head, and Radiation Efficiency for the Different Handset/Body Configurations Shown in Fig. 13. All Data is Computed at $915 \mathrm{MHz}$

\begin{tabular}{|c|c|c|c|c|c|c|}
\hline Configuratio & $\frac{P_{\text {abs }}^{\text {head }}}{P_{\text {del }}}$ & $\frac{P_{a b s}^{\text {hand }}}{P_{\text {del }}}$ & $\eta_{a}$ & $\frac{\mathrm{SAR}_{\max }^{\text {head }}}{P_{\text {del }}}$ & $\frac{S_{\mathrm{AR}}^{\text {hand }} \max }{P_{\text {del }}}$ & $\frac{\Delta A R_{\text {ave }}^{\text {head }}}{\dot{P}_{\text {del }}}$ \\
\hline \multicolumn{7}{|c|}{ Handset Rotated $60^{\circ}$ wuth Respect to Head } \\
\hline Monopole & 0.359 & 0.169 & 0.472 & 1.97 & 2.29 & 0.0875 \\
\hline Side Mounted PIFA & 0.382 & 0.301 & 0.317 & 3.81 & 4.54 & 0.0931 \\
\hline Side Mounted PIFA ${ }^{\dagger}$ & 0.212 & 0.318 & 0.470 & 3.14 & 5.42 & 0.1003 \\
\hline Back Mounted PIFA & 0.260 & 0.222 & 0.518 & 1.32 & 3.58 & 0.0634 \\
\hline \multicolumn{7}{|c|}{ Handset Upright with Respect to Head } \\
\hline Monopole & 0.351 & 0.184 & 0.465 & 2.06 & 2.43 & 0.0856 \\
\hline Side Mounted PIFA & 0.332 & 0.324 & 0.344 & 2.07 & 4.91 & 0.0809 \\
\hline Back Mounted PIFA & 0.258 & 0.225 & 0.517 & 0.90 & 3.53 & 0.0629 \\
\hline
\end{tabular}

${ }^{\dagger}$ Homogeneous spherical head $\left(\epsilon_{r}=58.5, \sigma=1.21\right)$

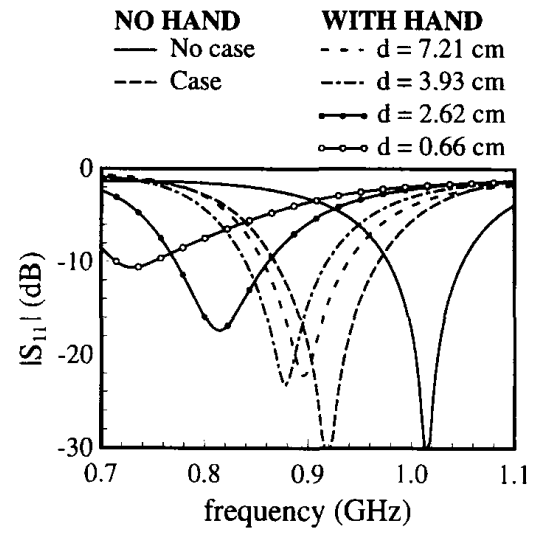

Fig. 11. Computed value of $\left|S_{11}\right|$ for the top-mounted BIFA on the handset without the plastic casing, with the casing, and with the casing and hand at four different locations.

measured data for the antenna with the hand absent and present at $d=6.56 \mathrm{~cm}$. This comparison shows good correlation between the experimentally and computationally obtained results.

The curves in Fig. 9 represent $\left|S_{11}\right|$ when the head and hand are present for two different head models. The curve labeled 'sphere' corresponds to the case of a homogeneous spherical ball of muscle $\left(\epsilon_{r}=58.5, \sigma=1.21 \mathrm{~S} / \mathrm{m}\right)$ with a radius of $9 \mathrm{~cm}$. The second head is the inhomogeneous model discussed in Section II-B. As can be seen, for this particular configuration the choice of models exercises little influence on the antenna input impedance. This insensitivity occurs because the input impedance is a reasonably local phenomenon which is influenced most significantly by structures in the near vicinity of the antenna rather than objects such as the head which are displaced somewhat from the antenna feed point. Once again, the measured data given in Fig. 9 shows that the FDTD accurately predicts the effects of the human operator on the antenna performance.

Unlike the input impedance, the radiation characteristics of the side-mounted PIFA are expected to show an increased sensitivity to the presence of the head as well as the physical model used. This expectation is confirmed in Fig. 10 which shows the radiation patterns normalized to the antenna gain at $915 \mathrm{MHz}$ when the handset is encased in plastic and rotated $60^{\circ}$ with respect to the head. The three sets of curves in Fig. 10 represent the patterns for the handset alone, the handset near the spherical head, and the handset near the detailed head $(b=1.97 \mathrm{~cm})$. When either head model is used, the hand is placed at $d=7.21 \mathrm{~cm}$. As anticipated, the antenna radiation pattern depends on the tissue presence as well as the choice of head models. Once again, the amount of power absorbed in the tissue is quantified in Table 2. As can be seen, the inhomogeneous head model results in somewhat higher absorption levels as compared to the simpler spherical head model for this configuration. This difference occurs because the sphere is physically smaller and has a permittivity/conductivity distribution which reduces the electromagnetic penetration depth as compared to the inhomogeneous model.

\section{Top-Mounted BIFA}

As mentioned above, proper antenna placement is a critically important issue when designing antennas for handheld devices. The goal is to find an antenna location to minimize radiation blockage by the hand. In general, observation reveals that the typical user will not hold the transceiver near the top. For this reason, the configuration shown in Fig. 1(c) is investigated. The variation of $\left|S_{11}\right|$ with frequency is shown in Fig. 11. The different curves 
Side-Mounted PIFA

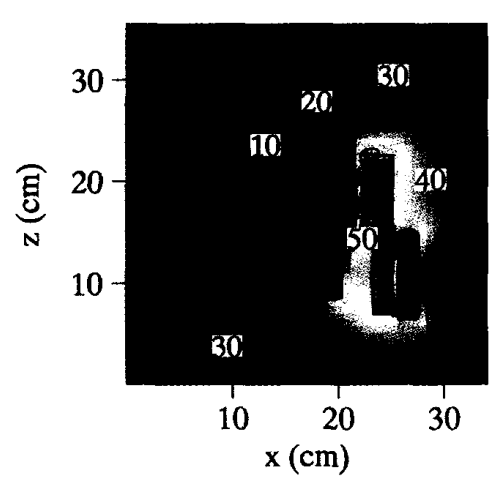

Monopole

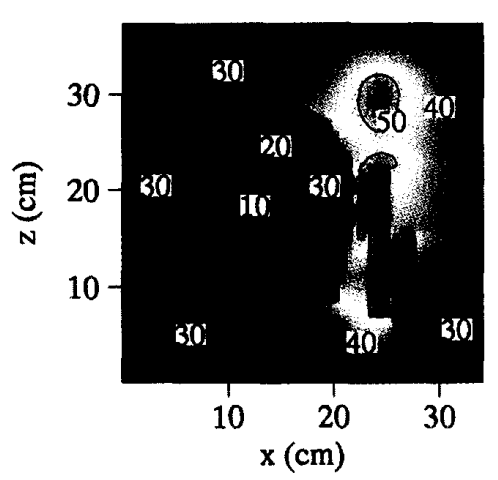

Side-Mounted PIFA (sphere)

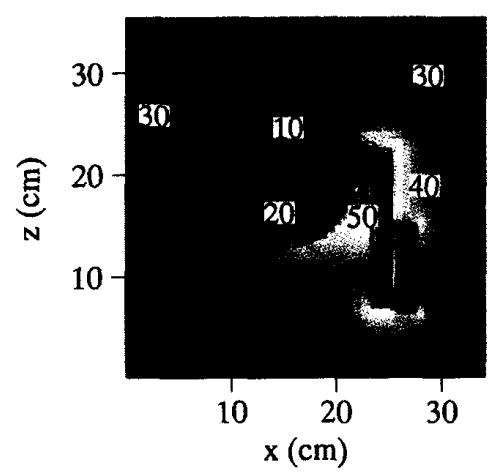

Back-Mounted PIFA

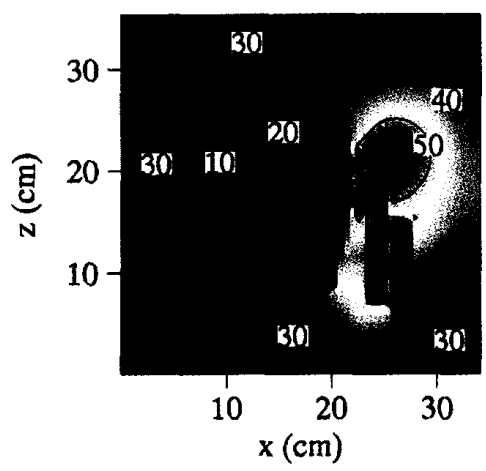

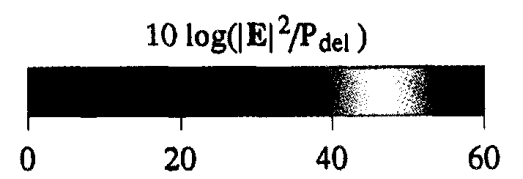

Fig. 12. Computed normalized near field distribution in a plane through the center of the head with $d=7.21 \mathrm{~cm}, b=1.97 \mathrm{~cm}$, and the plastic casing present at $915 \mathrm{MHz}$. The configurations are the side-mounted PIFA, the side-mounted PIFA with the spherical head, the monopole, and the back-mounted PIFA.

represent computations for the handset alone, with the casing, and with the casing and the hand at four vertically displaced positions. Consistent with the results for the sidemounted PIFA, the presence of the hand detunes the antenna resonance. However, in comparison with the geometry of Fig. 1(b), the degradation for the top-mounted BIFA is less severe for a given hand position. For this reason, configurations similar to this show potential as possible integrated antennas for hand-held transceiver units. The results in Fig. 11 also reveal the significant frequency detuning caused by the plastic casing. Compensation for this effect should be included in the integrated antenna design.

\section{Near Fields and Specific Absorption Rate}

Having investigated the effect of the body on the electromagnetic characteristics of these antenna structures, we now turn our focus to the field distribution and power absorption characteristics within the tissue. Fig. 12 compares the field variation around the plastic-covered handset, the head, and the hand at $915 \mathrm{MHz}$ for the side-mounted PIFA with the inhomogeneous and spherical head models and for the monopole antenna. Fig. 13 presents the SAR distribution for the same configurations. For each computation, $d=7.21$ $\mathrm{cm}, b=1.97 \mathrm{~cm}$, and the head is upright for simplicity in data presentation. The data plane is located at the center 

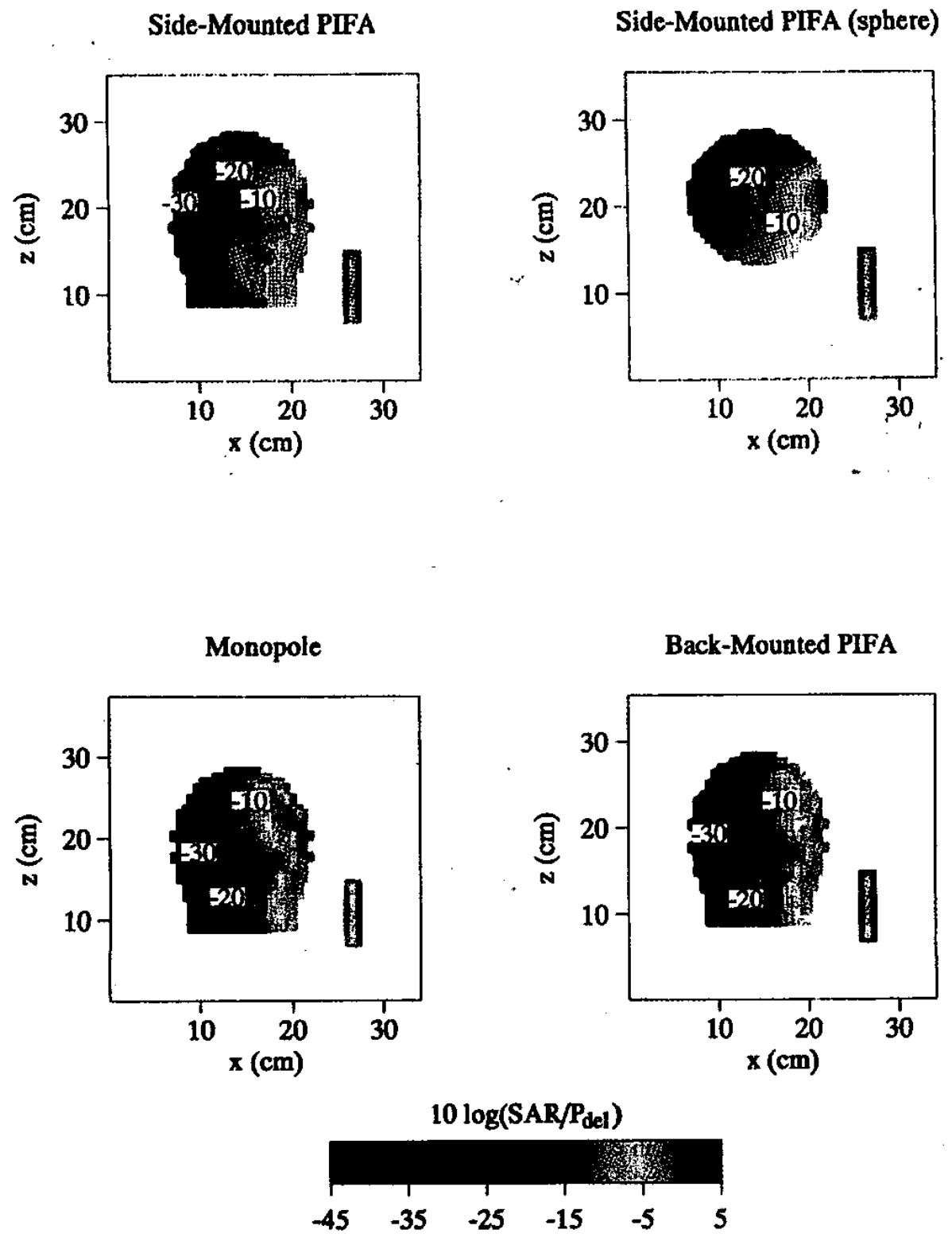

Fig. 13. Computed normalized SAR distribution in a plane through the center of the head with $d=7.21 \mathrm{~cm}, b=1.97 \mathrm{~cm}$, and the plastic casing present at $915 \mathrm{MHz}$. The configurations are the side-mounted PIFA, the side-mounted PIFA with the spherical head, the monopole, and the back-mounted PIFA.

of the head/handset combination, and the plots are viewed from the $-y$ direction (see Fig. 4). The values in Fig. 12 represent the total squared magnitude of the peak electric field per watt of total power delivered to the antentia; or-

$$
10 \log \frac{|\mathrm{E}|^{2}}{P_{\text {del }}} \text {. }
$$

Similarly, the values in Fig. 13 represent the SAR averaged over $1 \mathrm{~g}$ of tissue and normalized to the power delivered to the antenna using the expression

$$
10 \log \frac{\mathrm{SAR}}{P_{\text {del }}}
$$

Table 2 provides a numerical comparison of the peak SAR values occurring in the head and hand for each of the configurations in Fig. 12 and 13. The two sets of data correspond to the configurations where the handset is rotated $60^{\circ}$ and the handset is upright with respect to the head. As can be seen, each of the antenna structures results in very similar values of the peak SAR for both handset orientations with the exception of the side-mounted PIFA geometry. In this case, the rotated handset places the antenna nearly in contact with the ear tissue, resulting in a higher peak SAR as compared to the result for the upright handset. For all of the configurations, the peak 
SAR in the head occurs either in the ear tissue or in the skin/fat layer in the antenna vicinity. Table 2 also provides the SAR averaged over the entire head, which as expected is considerably lower than the peak SAR levels. The numbers in Table 2 and plots in Figs. 12 and 13 show that while the spherical model is useful for obtaining a first-order approximation to the effect of the head, an inhomogeneous model is required to obtain the detailed field variations in and around the tissue. It is important that the numbers provided here are consistent with previously reported measured and computed results [7], [8], [18]. Comparisons such as these are very useful for determining the suitability of different radiators for personal communications applications.

\section{E. Back-Mounted PIFA}

In an effort to reduce the exposure to the head, a PIFA element is mounted on the back of the handset, as shown in Fig. 1(d). Since the conducting handset chassis lies between the operator and the antenna, it is expected that the peak SAR in the head will be reduced. The resulting field and SAR plots are provided in Figs. 12 and 13, respectively. These results, coupled with the numerical results in Table 2 , serve to illustrate the substantial reduction in the peak SAR resulting from judicious placement of the element. Further refinement of similar antenna configurations may be possible to reduce the peak SAR and power absorption within the body.

The preceding results for radiation efficiency and peak SAR in the head have all been provided for a given separation between the head and the handset $(b=1.97 \mathrm{~cm})$. However, it is interesting to examine the effect of this distance on these parameters. Fig. 14 presents the variation of the antenna efficiency and peak SAR ( $1 \mathrm{~W}$ delivered power) in the head versus the distance $b$ for the monopole and the back-mounted PIFA configurations. As might be expected, the radiation efficiency increases with distance, while the peak SAR decreases in a nearly exponential fashion. It is noteworthy that as $b$ increases, the backmounted PIFA efficiency actually falls below that of the monopole. This phenomenon occurs because at these larger distances, the absorption in the hand becomes a more significant percentage of the total absorption. Since the power absorption in the hand induced by the PIFA is greater than that caused by the monopole (see Table 2), the PIFA efficiency is expected to fall below that of the monopole for large distances.

\section{CONCLUSION}

This paper has presented some key issues relating to the electromagnetic interaction between handset-mounted antennas and a human operator's biological tissue. The study has used the FDTD simulation technique in conjunction with detailed models of the antennas, handset, head, and hand. The FDTD implementation summarized in the paper has been made adequately flexible to accommodate modeling of a wide variety of antenna and tissue topologies.

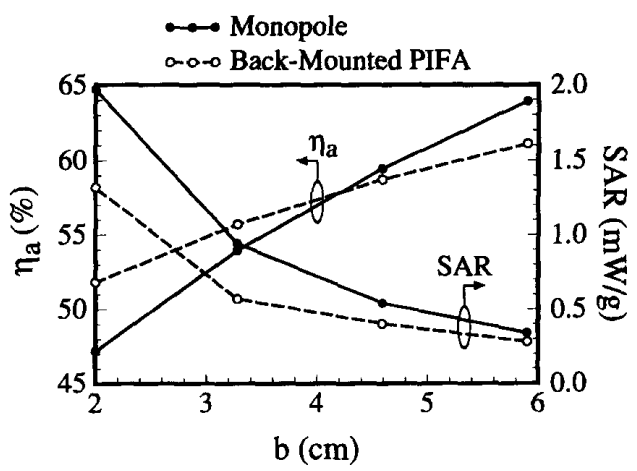

Fig. 14. Radiation efficiency $\eta_{a}(\%)$ and peak SAR in the head $\left(\boldsymbol{P}_{\mathrm{del}}=1 \mathrm{~W}\right)$ for the monopole and back-mounted PIFA versus the distance $b$ between the head and handset $(d=7.21 \mathrm{~cm})$.

The simulations presented for representative handset/tissue geometries have revealed that the tissue exercises a noticeable effect on the antenna input impedance, radiation patterns, and gain for both external and internal configurations. Most particularly, masking of low-profile antennas by the hand can result in serious detuning of the antenna impedance. In many cases, experimental results obtained at very low powers have been presented which verify the simulation accuracy and provide additional understanding into the antenna behavior. The numerical simulations also reveal that the SAR in the head has a peak between 2.0 and $3.8 \mathrm{~mW} / \mathrm{g}$ and an average between 0.08 and $0.10 \mathrm{~mW} / \mathrm{g}$ for $1 \mathrm{~W}$ of delivered power, and that the tissues absorb between 53 and $68 \%$ of the power delivered to the antenna for a head-handset separation of $2 \mathrm{~cm}$. It has been shown that by placing an internal antenna (PIFA) on the back of the handset away from the user, the peak and average SAR in the head and the power absorption may be reduced to $0.9 \mathrm{~mW} / \mathrm{g}, 0.06 \mathrm{~mW} / \mathrm{g}$, and $48 \%$, respectively.

\section{REFERENCES}

[1] J. J. C. Chang, R. A. Miska, and R. A. Shober, "Wireless systems and technologies: an overview," AT\&T Tech. J., vol. 72, pp. 11-18, July/Aug. 1993.

[2] K. S. Yee, "Numerical solution of initial boundary value problems involving Maxwell's equations in isotropic media," IEEE Trans. Ant. Propag., vol. AP-14, pp. 302-307, May 1966.

[3] A. Taflove and M. E. Brodwin, "Numerical solution of steady-state electromagnetic scattering problems using the time-dependent Maxwell's equations," IEEE Trans. Microwave Theory Tech., vol. MTT-23, pp. 623-630, Aug. 1975.

[4] R. Luebbers, L. Chen, T. Uno, and S. Adachi, "FDTD calculation of radiation patterns, impedance, and gain for a monopole antenna on a conducting box," IEEE Trans. Ant. Propag., vol. 40, pp. 1577-1583, Dec. 1992

[5] M. A. Jensen and Y. Rahmat-Samii, "Performance analysis of antennas for hand-held transceivers using FDTD," IEEE Trans. Ant. Propag., vol. 42, pp. 1106-1113, Aug. 1994.

[6] M. A. Jensen and Y. Rahmat-Samii, "FDTD analysis of PIFA diversity antennas on a hand-held transceiver unit," in 1993 IEEE AP-S Int. Symp. Dig., vol. 2, pp. 814-817, Ann Arbor, MI, June/July 1993.

[7] J. Toftgård, S. N. Hornsleth, and J. B. Andersen, "Effects on portable antennas of the presence of a person," IEEE Trans. Ant. Propag., vol. 41, pp. 739-746, June 1993.

PROCEEDINGS OF THE IEEE, VOL. 83, NO. 1, JANUARY 1995 
[8] P. J. Dimbylow, "FDTD calculations of the SAR for a dipole closely coupled to the head at $900 \mathrm{MHz}$ and $1.9 \mathrm{GHz}$," Phys. Med. Biol., vol. 38, pp. 361-368, Feb. 1993.

[9] P. J. Dimbylow and O. P. Gandhi, "Finite-difference timedomain calculations of SAR in a realistic heterogeneous model of the head for plane-wave exposure from $600 \mathrm{MHz}$ to $3 \mathrm{GHz}$," Phys. Med. Biol., vol. 36, pp. 1075-1089, Aug. 1991.

[10] D. M. Sullivan, O. P. Gandhi, and A. Taflove "Use of the finitedifference time-domain method for calculating $E M$ absorption in man models," IEEE Trans. Biomed. Eng., vol. 35, pp. 179-186, Mar. 1988.

[11] G. Mur, "Absorbing boundary conditions for the finitedifference approximation of the time-domain electromagnetic field equations," IEEE Trans. Electromagnetic Compat, vol. EMC-23, pp. 377-382, Nov. 1981.

[12] A. C. Eycleshymer and D. M. Schoemaker, A Cross-Section Anatomy. New York: Appleton-Century, 1911.

[13] D. M. Sullivan, "A frequency-dependent FDTD method for biological applications," IEEE Trans. Microwave Theory Tech., vol. 40, pp. 532-539, Mar. 1992.

[14] American National Standards Institute, IEEE C95.1-1991: IEEE Standard for Safety Levels with Respect to Human Exposure to Radio Frequency Electromagnetic Fields, $3 \mathrm{kHz}$ to $300 \mathrm{GHz}, 1991$, Piscataway, NJ 08855-1331.

[15] M. Fischetti, "The cellular phone scare," IEEE Spectrum, pp. 43-47, Mar. 1993.

[16] R. J. Luebbers and K. Kunz, "FDTD modeling of thin impedance sheets," IEEE Trans. Ant. Propag., vol. 40, pp. 349-351, Mar. 1992.

[17] T. Taga and K. Tsunekawa, "Performance analysis of a built-in planar inverted $F$ antenna for $800 \mathrm{MHz}$ band portable radio units," IEEE J. Selected Areas Commun., vol. SAC-35, pp. 921-929, June 1987.

[18] H. R. Chuang, "Human operator coupling effects on radiation characteristics of a portable communication dipole antenna," IEEE Trans. Ant. Propag., vol. 42, pp. 556-560, Apr. 1994.

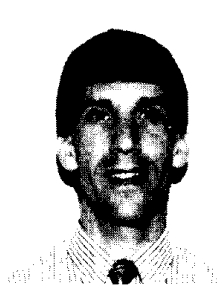

Michael A. Jensen (Student Member, IEEE) received the B.S. (summa cum laude) and M.S. degrees in electrical engineering from Brigham Young University (BYU) in 1990 and 1991, respectively. He is currently pursuing the $\mathrm{Ph} . \mathrm{D}$ in electrical engineering at the University of California, Los Angeles.

From 1989 to 1991 he was a graduate $\mathrm{Re}-$ search Assistant in the Lasers and Optics Laboratory at BYU. Since 1991, he has been with the Antenna Laboratory at UCLA as a graduate student researcher. His main research interests include radiation and propagation for personal communications, numerical electromagnetics, optical fiber communication, and implementation of finite-difference schemes on massively parallel computer architectures.

Mr. Jensen is a member of Eta Kappa Nu and Tau Beta Pi. In 1990 he received a National Science Foundation Graduate Fellowship.

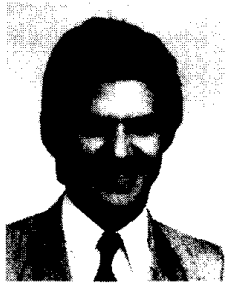

Yahya Rahmat-Samii (Fellow, IEEE) received the M.S. and Ph.D. degrees in electrical engineering from the University of Illinois at Champaign-Urbana.

$\mathrm{He}$ is a Professor of Electrical Engineering at the University of California, Los Angeles, and was a Guest Professor at the Technical University of Denmark (TUD) in 1986. He has also been a Senior Research Scientist at the NASA Jet Propulsion Laboratory/California Institute of Technology since 1978, where he has contributed to the advancement of antenna technology for space programs. $\mathrm{He}$ has made pioneering contributions to the developments of near-field plane-polar and bipolar antenna measurements, microwave holographic diagnostics, mobile satellite communication antennas, reflector surface compensation, multireflector antenna diffraction analysis and synthesis, scattering and radiation from complex objects, RCS computations, singularity in dyadic Green's function, high power microwave (HPM) antennas, EMP and aperture penetration, the spectral theory of diffraction (STD), and GTD. He has authored or coauthored over 300 technical journal articles and conference papers and has written chapters in 13 books.

Dr. Rahmat-Samii became a Fellow of IAE in 1986 and was the 1984 recipient of the Henry Booker Award of URSI. He was appointed an IEEE Antennas and Propagation Society Distinguished Lecturer and presented lectures internationally. He was an elected IEEE AP-S AdCom member for the second term and has been an Associate Editor of the IEEE Transactions on Antennas and Propagation and the society's Magazine $\mathrm{He}$ is currently the elected President of IEEE AP-S. He was the Chairman of the IEEE Antennas and Propagation Society of Los Angeles during 1987-1989, which received the Antennas and Propagation Best Chapter Award from the AP Society in 1989. He is one of the three international Editors of the IEE book series on electromagnetics and antennas. He is also one of the Editors of the Journal of Electromagnetic Waves and Applications. He is a Director of AMTA (Antenna Measurements Technique Association) and the Electromagnetics Society. He is listed in Who's Who in America, Who's Who in Frontiers of Science and Technology, and Who's Who in Engineering. He has received numerous NASA Certificate of Recognitions and recently earned the JPL Team NASA's Distinguished Group Achievement Award. In 1992, he was the recipient of the Best Application Paper Award (Wheeler Award) for a paper published in the Transactions in 1991. He is a member of Commissions A, B, and J of USNC/URSI, Sigma Xi, Eta Kappa Nu, and the Electromagnetics Academy. 